\title{
Several Considerations on Test Method of Helium Mass Spectrometer Leakage Detection
}

\author{
Henan Four Construction Co., Ltd. Kaifeng 475000, Henan
}

\section{Introduction}

For evaluation of a spacecraft quality, leak tightness of the spacecraft is a key factor, which is directly related with operation in the normal trajectory and spacecraft launch. Even a tiny loophole may cause great losses. Therefore, test to the leak tightness performance should be well addressed during spacecraft research. ${ }^{1}$ At present, there are following approaches regarding to leak tightness of a spacecraft: helium hood method, helium mass spectrometer vacuum method, non-vacuum leakage detection method, sniffing probe leakage detection method, and among others, depending on different application scenarios. For example: for the aerospace electronic components, the helium hood method is mainly adopted for leak tightness test; for the aerospace components and assemblies, helium mass spectrometer vacuum method would be used. The test method may vary, but they share the same helium mass spectrometer leakage detector, which is the critical equipment in leak tightness check, specifically for the study on the linearity of input and output of the detector.

However, few scholars have conducted quantitative analysis on linearity of the leak detector so far. Although some researchers conducted research on linearity of the leak detector, they mainly analyzed helium concentration in the leak detector, and changed value output of leak detector by changing partial pressure of helium in the mass spectrum chamber. $^{2}$ In contrast to the outdated method, a new approach will be discussed in the paper to facilitate the research into linearity between inlet pressure and output reading in the leak detector.

\section{Theoretical analysis on the test methodology}

The main operation principle of the helium mass spectrometer leak detector is to ionize some mixed gas in the mass spectrum chamber, so as to generate a kind of ion with energy. These ions have different mass-tocharge ratios, therefore, they have different moving trajectories in the analyzer before separation. ${ }^{3}$ Then helium ions shall be independently collected until it is possible to magnifying them for display. The general helium mass spectrometer leak detector has two specifications, namely DC helium mass spectrometer leak detector and most widely used counter current mass spectrometer leak detector. The operational principle of such leak detector is based on the compression ratio between the bled air rate and vacuum pump. If the related gas mass is very small, the corresponding compression ratio is also relatively small. Meanwhile, there will be more and more reverse diffusion molecules in the gas. Once these reverse diffusion molecules are spread to the mass spectrum chamber, the output value will appear on the leak detector. The paper mainly focuses on Phoenix L300 counter current helium mass

\begin{abstract}
In the research into helium mass spectrometer leakage detection, a method for linearizability investigation has been put forward recently. Meanwhile, it has been demonstrated the rationality of the method through multiple tests based on the study in the principle of constant pumping speed of the mechanical pump in vacuum. The method realizes the control over the pressure through adjusting the leak detector inlet, so as to adjust gas flow rate in the leak detector. The concentration of helium in mixed gas is controlled via changing helium flow rate during leakage detection, so as to influence value display of the leak detector. With regard to test of linearity of the leak detector, a research has been conducted into relationship between the inlet pressure and output readings. The methodology of the research is characterized by simplicity, convenience, cost effective, and its test range covers most of the test leakageage rates in practice.
\end{abstract}

Key words: Helium Mass

Spectrometer; Leakage Detection; Linear Method

Published online: 30th Sept, 2017

spectrometer leak detector from Leybold Company. (which finds its wide application in various 
environments and modes for its portable cutting edge design and proven quality.)

Refer to Figure 1 for operational principle of the leak detector. It mainly conducts leakage detection through Gross mode. Suppose that the inlet pressure of the leak detector is $P_{1>20 \mathrm{~Pa}}$ (the threshold between two motion modes of Fine

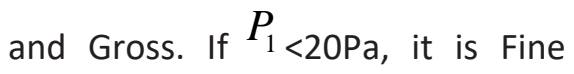

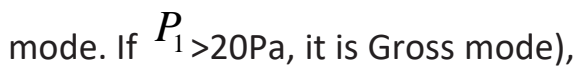
the Groos mode shall be initiated to conduct leakage detection, and the valves marked with 3, 4, 5 and 6 in the figure are at the closed state, and the rest are at the open state; if the leak detector is in Fine mode, the valves marked with 1,3 ,
$4,5,6 / 2 b$ in the figure are closed, and the rest are at the open state. It can be seen that the leak detector in the two states are different in terms of internal hardware used. Among the values of the leak detector, the pumping speed, compression ratio and other values are different. Therefore, there is no comparability in two modes. ${ }^{4-6}$

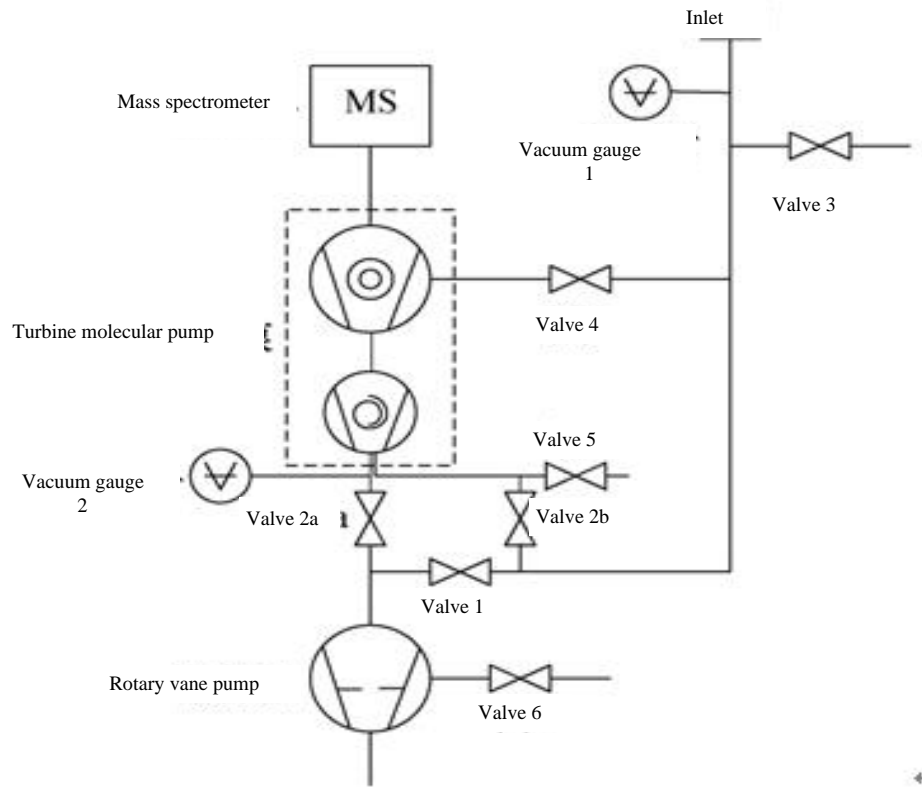

Figure 1 Schematic diagram of L300 helium mass spectrometer leak detector 
If it is not in the vacuum, leakage detection in Gross mode shall be adopted due to higher pressure. If the mixed gas flow in the leak detector is $Q=P_{1} S_{p}(1)$, where Q represents the total flow of the leak detector; $S_{p}$ represents the pumping speed at the inlet of the leak detector. The mixed gas with related helium concentration $\gamma$ represents that the helium flow entering the leak detector is

$G_{\mathrm{He}}$

Where,

$G_{H e}=Q \gamma=p_{1} S_{p} \gamma$

Meanwhile, the pumping speed in the inlet of the leak detector is mainly provided by the mechanical pump. Existence of the molecular pump in the leak detector mainly changes vacuum degree in mass spectrum chamber to the level at work. If the pumping speed in the mechanical pump is larger than 30Pa inlet pressure, its speed will be constant. ${ }^{7}$ For variation relationship diagram of the pumping speed and inlet pressure of the rotary vane pump with model of D2.5E, shown as Figure 2.

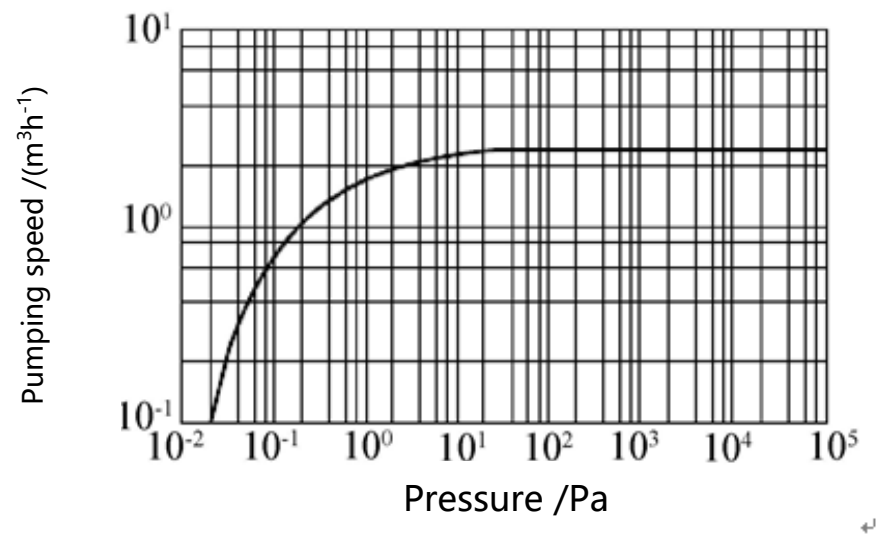

Figure 2 Relationship diagram of inlet pressure and pumping speed of the rotary vane pump 
Therefore, if the inlet pressure $P_{1}>30 \mathrm{~Pa}$, the pumping speed of the mechanical pump is maintained unchanged with continuous change in the inlet pressure, the result value obtained from Formula (2) will be constant. Relationship between the output value of the leak detector and the incoming helium amount can be expressed as I=k $G_{H e}$ (3). Where, I am the value output by the leak detector; $k$ is the value of linear performance in the leak detector. Combine Formula (2) with Formula (3) to obtain I=k $P_{1}$ $S_{p} \gamma$ (4). With regard to other mixed gases in helium, If $\alpha=k S_{p} \gamma$, then $\mathrm{I}=\alpha P_{1}$ (5).
Therefore, when L300 leak detector is used, suppose that the concentration of mixed gas in the leak detector is constant, if the inlet pressure is larger than 30pa, there will be proportional relationship between the inlet pressure of leak detector $P_{1}$ and the output value I of the leak detector. ${ }^{5}$ That is to say, if Formula (5) is reasonable, then Formula (3) will be reasonable.

\section{Method verification}

The paper will verify the correctness of Formula (5) through a series of tests ${ }^{6}$. First, a test will be conducted in a relatively closed environment with slow air flow and constant temperature, such as in the tooling room for test, general assembly and experiment of the spacecraft.
The output value will be checked by adjusting the inlet pressure of the leak detector. The inlet and outlet pressure values in the leak detector are then compared, suppose that the helium volume ratio in the atmosphere is $5 \times 10^{-6}$, and other mixed gases are uniformly distributed. To ensure the constant helium concentration in the air, a closed space with slow air flow shall be needed to guarantee the higher accurate test results. A test under Cross mode of leak detector has been conducted, and the relationship diagram between the output value I and $P_{1}$ inlet pressure is as shown in Figure 3.

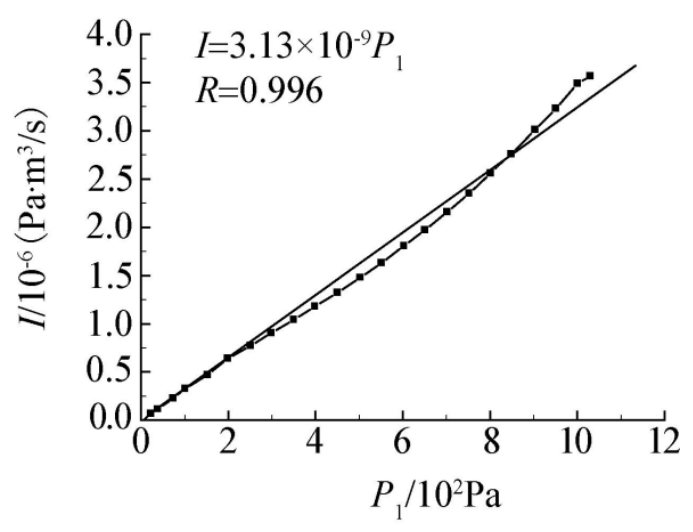

Figure 3 Relationship curve between $P_{1}$ and I 
It can be seen from Figure 3 that the relationship between the output value and inlet pressure of the leak detector is linear. Moreover, the linear fitting coefficient value $R$ (it is the variation percentage between independent variable and dependent variable. The value closer to 1 , the better the linearity) is very close to 1 .
Therefore, L300 leak detector in Gross mode has good linearity. It can be observed from Figure 3 that L300 leak detector in Gross mode has good linearity in the range from $1 \times 10-7$ to $1 \times 10-6 \mathrm{~Pa} \mathrm{~m} 3 / \mathrm{s}$. It is the exact the range we focus. ${ }^{8-10}$

Firstly, it has to be pointed out that the data obtained by each experimenter has good repeatability.
Therefore, the related curve diagram has not been given here. Meanwhile, it also indicates a common experiment phenomenon. Moreover, $\alpha$ values obtained by each experimenter at different times are relatively close, which means that the value is constant. Refer to the value in Table 1, it has been proven through tests the accuracy of Formula (5).

\begin{tabular}{|c|c|c|c|}
\hline Time & $\begin{array}{c}\alpha \text { value } /\left(\mathrm{m}^{3} \cdot \mathrm{s}^{-}\right. \\
1)\end{array}$ & $\begin{array}{c}\text { Average } \\
\text { value } /\left(\mathrm{m}^{3} \cdot \mathrm{s}^{-1}\right)\end{array}$ & Error \\
\hline 1 & $3.13 \times 10^{-9}$ & \multirow{5}{*}{$3.25 \times 10^{-9}$} & $-3.69 \%$ \\
\hline 2 & $3.31 \times 10^{-9}$ & & $1.85 \%$ \\
\hline 3 & $3.29 \times 10^{-9}$ & & $1.23 \%$ \\
\hline 4 & $3.28 \times 10^{-9}$ & & $1.00 \%$ \\
\hline 5 & $3.24 \times 10^{-9}$ & & $-0.31 \%$ \\
\hline
\end{tabular}

Table $1 \alpha$ values and errors at different times

It can be obtained from Formula (3) that if the detected gas concentration is constant, $\alpha$ value is related with the value of the leak detector only. Therefore, it can be concluded that the essential property of the leak detector can be expressed by value $\alpha$.

\section{Conclusion}

The theoretical analysis regarding leak detector has been conducted in the paper, together with the corresponding experiment, and linear relationship between the output value of the leak detector and the inlet pressure is analyzed in details, which has modified the previous method to detect gas concentration by detecting linear relationship of the leak detector.

\section{References}

[1] An Qi, Luo Xiaoyu. Analysis of Leakage Test with Helium Mass Spectrometer [J]. Electronic Product Reliability and Environmental Testing, 2014,32(4):34-38.

[2] Bai Jinhao, Wan Cong. Key Points in Process Method and Detection of Helium Mass Spectrometer Leakage Detection [J]. Speed Reading, 2016(6).
[3] Jin Yuquan. Study on the Failure Criteria for Helium Mass Spectrum Fine Leakage in the Standards [J]. Electronic Product Reliability and Environmental Testing, 29(3):1-3.

[4] Wang Xiaogang. Helium mass spectrometry leak detection in the application of air seal detection [A]. China Aviation Society aviation maintenance engineering branch. Aviation equipment maintenance technology and application of Proceedings [C]. China Aviation Society aviation maintenance works Professional club: 2015: 5.

Development of Comprehensive Performance Test Device for High Temperature Seal Gasket [D]. East China University of Science and Technology, 2015.

[6] Liu Zhidong. High pressure and vacuum leakage rate of the $[A]$. Chinese Academy of Sciences Beijing Science Instrument Development Center. The second national vacuum leak detection technology industry application papers [C]. Chinese Academy of Sciences Beijing Science Instrument Development Center: , 2000: 3.
[7] Yimei Xiu, Yan Li. The sealing test of arrester - helium mass spectrometry leak detection method [J]. China Electric Porcelain, 1982, (02): 29-34. [2017-0920] .OOI: 10.16188 / j.isa.10038337.1982.02.009

[8] Hao Chunlei, Qin Ling, Zhang Liang, Liu Shuang.Application of helium leaking technology in the manufacture of urea synthesis tower [J]. Pressure vessel, 2006, (09): 35-37 + 20. [2017-09- 20].

[9] Su Liping, Zhou Weiming.Discussion on the Method of Leakage Detection of Vacuum Vessel - Taking the Leak Detection Technique of Helium Mass Spectrometry Instead of the Traditional Leak Detection Method [J]. Chemical Equipment Technology, 2004, (02): 3738. [2017-09 -20] .DOI: 10.16759 / j.cnki.issn.1007-7251.2004.02.013

[10]. Electronic Standardization and Quality, 2000, (06): 31-33 + 40. [201709-20]. [J]. Electronic Standardization and Quality, 2000, (06): 31-33 + 40 . [2017-09-20]. 